\title{
Contemporary echographic characteristics in diagnosis of abnormal placental invasion
}

\begin{abstract}
Objective: The main reason of placenta accrete nowadays is the increase in the number of repeated cesarean sections. The aim of our study was to assess the possibilities of ultrasound in placenta accreta diagnostics, to verify the echographic signs of PA in placenta praevia and to compare and analyse our results with Medline findings.
\end{abstract}

Material: 162 pregnant women with non-migrating placenta praevia and at high risk of placenta accreta were examined in the sonographic department from 2006 to 2011. Several echographic markers, allowed to suspect invasion of placental tissue into the myometrium, were noted. When PA was suspected, MRI was performed and than morphologic examination verified our diagnosis

Results: Several echographicmarkers were revealed: lacunae, intensive collateral vasularization, bulging of placenta praevia and thinning of hypoechogenic zone of the myometrium and others.MR imaging was conducted in 9 cases of suspected placenta increta and was verified in 8 cases. C-section and hysterectomy were performed in 13 of 18 cases. No internal injuries of pelvic organs were revealed.

Conclusion: Echopraphy is a valuable non-invasive method in the group with placenta praevia, it allows to verify the placental invasion into the myometrium and to exclude this pathology. Obtaining the results of these examination will be helpful in reducing the maternal morbidity and mortality in high-risk group.

Keywords: antenatal care and diagnosis, high-risk pregnancy, medical and surgical complications of pregnancy, labour, delivery
Volume I Issue 2 - 2015

\author{
BoykovaYu V, Eremina OV, Ejova LS, \\ Kulabuhova EA, Gus Al \\ Ultrasound, MRI and Morphology departments, VI Kulakov \\ Federal Research Center for Obstetrics and gynecology, Russia
}

Correspondence: Eremina OV,VI Kulakov Federal Research Center for Obstetrics and gynecology, ultrasound, MRI and morphology departments, Academician Oparina str,4, Moscow, Russia, I 17997,Tel 79104549169, Email olga.erem@gmail.com

Received: October 06,2015 | Published: October 28, 2015
Abbreviations: AP, abnormal placentation; GA, gestational age; PPV, positive predictive value; NPV, negative predictive value

\section{Introduction}

The incidence of placenta accreta in the past 30 years has increased significantly. The main reason for this is the increase in the number of repeated cesarean sections. Accurate and timely diagnosis of abnormal placentation is crucial for the selection of pregnancy surveillance type and delivery method.

Abnormal placental invasion encompasses penetration of chorionic villi into the myometrium within the bounds of decidua basalis. All types of abnormal placentation (AP) make difficult the placental removal after delivery. ${ }^{1}$ Predisposing factors of AP are: placenta praevia, implantation of a gestational sac in the isthmus, repeated cesarean sections and other uterine operations, recurrent miscarriage, chronic atrophic endometritis. It is more often in multiparous women, older than $35 .^{2-5}$ There are 3 main types of AP, they are classified according to the depth of placental invasion. In placenta accreta chorionic villi are attached to the myometrium, without penetrating the uterine walls. PA is diagnosed during delivery. In this case no hysterectomy is needed. ${ }^{2}$ Placenta increta and percreta are associated with the penetration into the myometrium, consequently it causes severe complications during C-section: massive haemorrhage and DIC syndrome, urether and bladder damaging, amniotic embolism, polyorganic insuffiency and death. ${ }^{2-6}$

In the past 10years due to a large amount of repeated C-section, the incidence of placenta accreta grew significantly, therefore the number of hysterectomy also increased and life quality after several operations and due to massive bleedings decreased. At the same time echographic and MR imaging of this pathology are developing. ${ }^{6,7}$ When placenta accretais diagnosed before delivery, a multidisciplinary approach may improve patient outcome. This approach includes presence of skilled obstetricians, anesthesilogists and midwifes. Also well-equiped operating room, blood products, hemostatic drugs and cell-saver technology are needed. ${ }^{2,8}$ Thereby, abnormal placental invasion is still a diagnostic challenge. For these reasons, improving diagnosis of placenta accrete and its invasion into myometrium and other organs of pelvic floor is absolutely necessary for clinicians. Despite of large amount of research and scientific articles about AP, the prenatal diagnosis of this serious pathology is still a challenge for clinicians. The aim of our study was to assess the possibilities of ultrasound in placenta accreta diagnostics, to verify the echographic signs of PA in placenta praevia and to compare and analyse our results with Medline findings.

\section{Materials and methods}

162 pregnant women with non-migrating placenta praevia and at high risk of placenta accreta were examined in the sonographic department from 2006 to 2011.2 patients with gestational sac in the cesarean scar, who refused of abortion, were also included in the research group. Transabdominal, transvaginal sonography and color Doppler were conducted in each patient. An echography was performed from 7 to 39 weeks of gestational age (GA), once a month. All patients were included in the research after 28 weeks of GA. We noted several echographic markers, allowed to suspect invasion of placental tissue into the myometrium: 
i. Enlarged intervillous lacunae in the maternal surface with turbulent blood flow (Figure 1).

ii. Uneven, bumpy boundaries of lamina basalis of maternal placenta.

iii. Uterine bulging and deformation.

iv. An intensive blood flow in collateral uterine vessels.

v. A significant thinning of the myometrium of placental bed in the anterior and posterior uterine wall.

vi. Presence of the placental tissue in the cervix and lower uterine segment.

vii. A significant thickening of placenta praevia in the isthmus (Figure 2).

viii. Echographic markers of non-accreta placenta were plane surface between maternal part of the placenta and myometrium, evenness of placenta and the absence of intervillous lacunae with turbulent blood flow in the maternal surface of placenta.

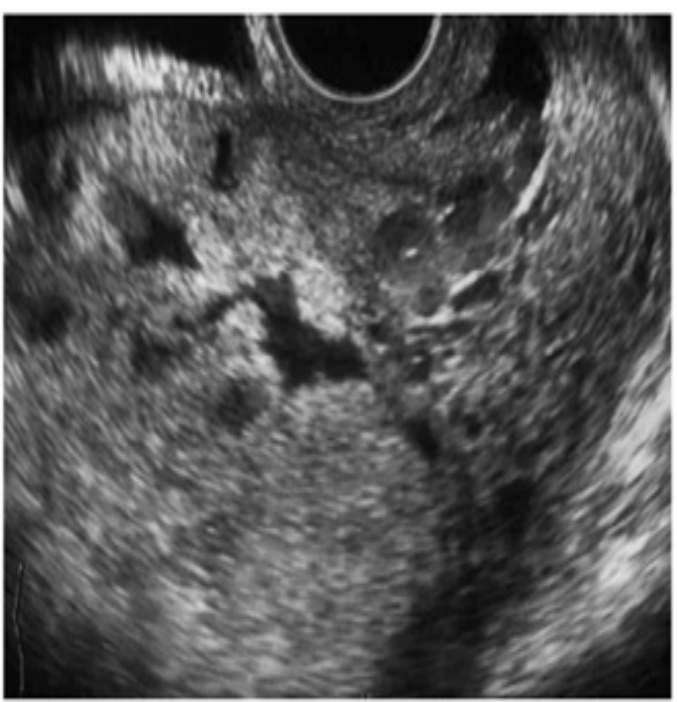

\section{$337 \times 259 \mathrm{~mm}(72 \times 72$ DPI)}

Figure I Maternal surface of placenta.

When PA was suspected, pelvic MRI was conducted. While using MRI, T2 weighted images were obtained in three planes (sagittal, axial and coronal). The variety of section thickness was $0,3-0,6 \mathrm{sm}$ and the vision fields-32-42sm. MR imaging showed a diffuse heterogeneity of placental parenchyma, uneven lacunar dilatation, Uneven, bumpy boundaries of maternal placenta, bulging of placenta with parallel thinning or defects of abutting myometrium surface and also an uterine wall deformation. Intraoperative and histopathologic verification of our diagnosis were performed. Morphologic analysis consisted of macroscopic visualisation of the removed uterus and microscopic examination of paraffin sections, colored by hematoxylin-eosin and picrotoxin (Van Gison method). To assess the possibilities of imaging diagnostics of PA, 162 multipara patients (18-42years, 27,7avg) were examined. Single C-section was performed in 16 cases, repeated (2) was conducted in 7 and third C-section was in 2 cases. Are current miscarriage occurred in 104/162 patients Statistical analysis was performed using double diagnostic test and calculating specificity, sensitivity, positive and negative predictive value.

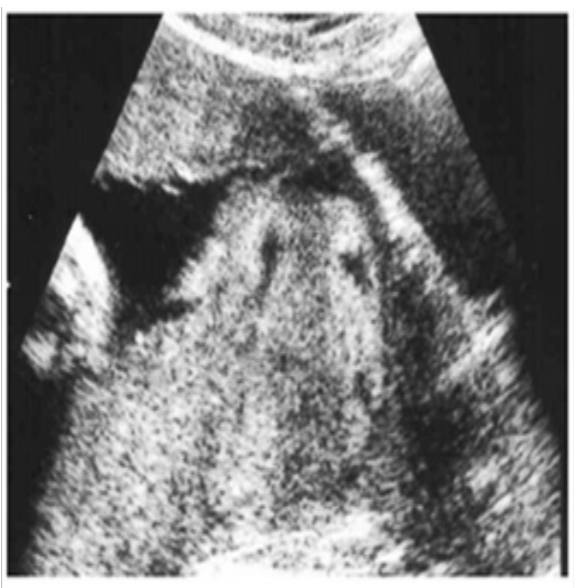

$194 \times 147 \mathrm{~mm}(96 \times 96091)$

Figure 2 Thickening of the isthmus.

\section{Results}

162patients with non-migrating placenta praevia were examined, in 18 cases at $28-36$ weeks of gestational age PA was suspected. The placental bed was in the anterior and posterior uterine walls. After 28 weeks all seven echographic markers were visualized in 9cases. Patients with gestational sac in the C-section scar were also in this group. In 5cases four markers were revealed: lacunae, intensive collateral vasularization, bulging of placenta praevia and thinning of hypoechogenic zone of the myometrium. In 4 cases only lacunar dilatation and uneven contour of maternal placental surface were noted.

MR imaging was conducted in 9cases of suspected placenta increta. In 8 of 9 cases placenta increta was verified. C-section and hysterectomy were performed in 13 of 18 cases. No internal injuries of pelvic organs were revealed. PA was confirmed in 14patients, in 13 women after morphologic examination of the uterus and placenta and in 1-during the postpartum period. In last case after the delivery the large part of placenta was removed and the tissue of invaded placenta praevia was revealed during screening sonography and MRI in postpartum. This tissue was invaded in $2 / 3$ of the uterine cavity. We refused of hysterectomy because of the absence of life-threatening haemorrhage and the obstinate desire of the woman. The woman is regularly examined by our colleagues.

The macroscopic analysis of the uterus showed that its size was from $14 * 9 * 14 \mathrm{sm}$ to $16 * 10 * 15 \mathrm{sm}$, the middle thickness of the uterine wall was $4-4,5 \mathrm{sm}$ (Figure 3 ). The pathologist visualized a flabby, anemic uterus with a 7-8sm incision in the lower uterine segment. In the anterior wall of the 4uterus were scars from previous $\mathrm{C}$-sections. The anterior placentation was noted in 8uterus and posterior-in 5. The microscopic examination in 9cases verified a partial placenta accrete, the chorionic villi were attached to the submucous layer of the myometrium, no decidual membrane was viewed. In these zones the pathologists confirmed edema, garneting, mucoid swelling, necrosis and gialinosis of the muscle fibers. Placenta increta was diagnosed in 4 cases, chorionic villi invaded muscle fibers $0.2-0.3 \mathrm{sm}$ beneath the surface.

In histologic sections muscle tissue was characterized by dilated plethoric blood vessels and haemorrhage areas. Dystrophic changes 
and fibrinoid accumulation were noted in the chorionic villi and cytotrophoblast were. No signs of chorionic epithelium proliferation were diagnosed during the microscopy. In 4 of 18 cases placenta accrete was not verified, therefore in the group of patients with non-accreta placenta praevia $(\mathrm{n}=144)$, in 5 women PA was diagnosed during C-section, but hysterectomy was performed only in 3patients. In the rest 2operations deep curettage of the uterine cavity was conducted.

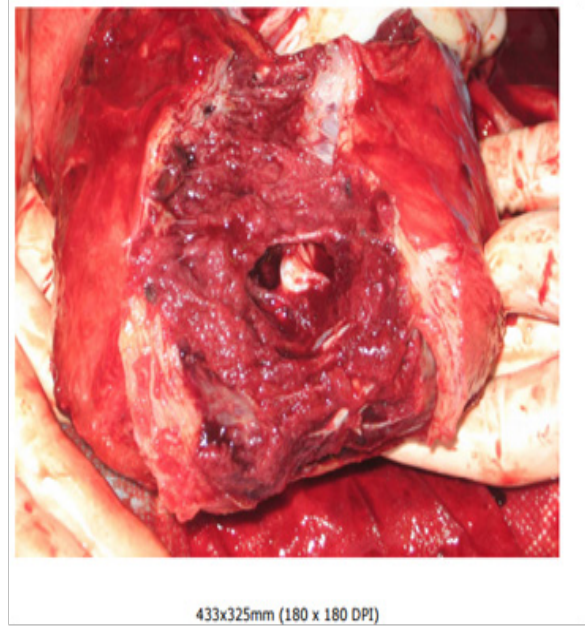

Figure 3 Uterine wall.

Finally, the echographic sensitivity and specificity in detecting PA were $74,0 \%(14 / 19)$ and $97,2 \%(139 / 143)$ with a false-positive rate $2,8 \%$. Positive predictive value (PPV) was 78,0\% (14/18) and negative predictive value (NPV) $-96,5 \%(139 / 144)$.
Placenta accrete in placenta praevia group occurred in $11,7 \%$. Hysterectomy was made in $72 \%$ in increta group, while in placenta praevia group-only in $2 \%$.Haemorrage volume in the group with placenta increta varied from 2500 to $7500 \mathrm{ml}$, mean volume was $3400 \mathrm{ml}$, while in the second group it was $900-4000 \mathrm{ml}$ (mean volume $1200 \mathrm{ml}$ ). 34children were delivered at $28-34$ weeks and $128-$ at $37-39$ weeks. No neonatal seizures were revealed in all 162 deliveries.

\section{Discussion}

In last decades there is an increasing interest in diagnosis placenta praevia increta. It is very important to verify timely and precise diagnosis and to take it into consideration for planning place and date of delivery, to organize an expert team of surgeons and anesthesiologist, to prepare blood products. ${ }^{2,4,6}$ Nowadays, in Russia and other countries echography is the primary diagnostic tool for PA diagnosis before delivery. According to literature, its predictive value is about $80 \%, 4,6,9,10$

Nevertheless there are different data about specifity and sensitivity of this method in verifying placenta accrete. ${ }^{8,10-12}$ The results of our research $(n=162)$ show, that the sensitivity of ultrasound was $74,0 \%$, and specificity-97,2\%. The identical research (Table 1) reveal, that sensitivity of US varies from 77,0 to $94,0 \%$. Our work showed a lower result because of the presence of 4 patients with doubtful echographic features ( 2 markers of abnormal placental invasion). Comstock ${ }^{9}$ supposed, that thinning of the myometrial hypoechogenic zone is not a reliable marker, because it can be visualized in normal placenta, localized in the anterior wall. ${ }^{9}$ Shih et al. ${ }^{13}$ showed that there were false positive markers in the group with single marker of PA. ${ }^{13}$ Therefore the sensitivity was maximal $(100 \%)$ in the presence of all seven echographic signs of PA.

Table I Summary of binary diagnostic test results of our study compared to other authors

\begin{tabular}{lllll}
\hline Study & Sensitivity, \% & Specificity, \% & PPV, \% & NPV,\% \\
\hline Our study $(\mathrm{n}=162)$ & 74,0 & 97,2 & 78,0 & 96,0 \\
Esakoffet al. $(\mathrm{n}=108)$ & 89,5 & 91,0 & 68,0 & 97,6 \\
Chou et al. $(\mathrm{n}=80)$ & 82,4 & 96,8 & 87,5 & 95,3 \\
Warsak et al. $(\mathrm{n}=453)$ & 77,0 & 96,0 & 65,0 & 98,0 \\
Dwyer et al. $(\mathrm{n}=32)$ & 93,0 & 71,0 & 74,0 & 92,0 \\
\hline
\end{tabular}

Specificity of the method in our work was higher, comparing with the others, ${ }^{8,10-12}$ because there were the exact exclusion criteria in PA group. An even contour between maternal placental surface and myometrium, evenness of the thickness, an absence of intervillous lacunae with the turbulent blood flow-all these data indicated nonaccreta placenta praevia even in the group with the scars after repeated $\mathrm{C}$-sections and the anterior placentation. The positive predictive value (PPV) was $78,0 \%$. Our data are comparable to Dwyer et al. ${ }^{11}$ The negative predictive value (NPV) in our research also showed a high reliability of US in excluding placenta accreta, similar data are seen in the other publications. ${ }^{8,10-12}$ It is very important to assess the group of the patients without placenta accrete, it is necessary for delivery planning. The retrospective analysis of ultrasound video scanning, performed in the group with verified placenta accrete, revealed several US features of this pathology. According to this research, the main echographic markers of PA are: enlarged intervillous lacunae in the maternal surface with turbulent blood flow, uneven, bumpy boundaries of maternal placenta, uterine bulging and deformation, a significant thinning of the hypoechogenic zone of myometrium in the cervix and lower uterine segment.

Therefore an intensive blood flow in collateral uterine vessels, significant thinning of the hypoechogenic zone of myometrium and a thickening of placenta praevia were revealed in non-confirmed and in verified cases of PA. The majority of the received data are the similar to other publications. ${ }^{2,7,14-16}$ But some authors indicate an important role of intensive collateral vascularization. ${ }^{7,13,17}$ Shih et al., ${ }^{13}$ showed a high sensitivity (97\%) and specificity (92\%) of Color Doppler Imaging in diagnosis coherent vessels between t. serosa of the uterus and the bladder wall.

Although an application of ultrasound remains a primary modality in assessment of placental implantation, the usage of MRI allows to improve the reliability of diagnosis. Baughman ${ }^{2}$ noted, that there are few studies in literature, which examined the specific MR feature of placenta accrete. ${ }^{2}$ 
To conclude, echography is a valuable non-invasive method in the group with placenta praevia, it allows to verify the placental invasion into the myometrium and to exclude this pathology. A routine examination should include the transabdominal, transvaginal sonography and color Doppler of placental tissue and placental bed and MRI of the uterus. Obtaining the results of these examination will be helpful in reducing the maternal morbidity and mortality in high-risk group.

\section{Acknowledgements}

None.

\section{Conflict of interest}

The author declares no conflict of interest.

\section{References}

1. Benirschke K, Kaufmann P. Pathology of the human placenta. Springer-Verlag. 2006;457:460-462.

2. Baughman WC, Corteville JE, Shah RR. Placenta accreta: spectrum of US and MR imaging findings. RadioGraphics. 2008;28(7):1905-1916.

3. ACOG Committee on Obstetric Practice. ACOG Committee opinion no. 266, January 2002: Placenta accreta. Obstet Gynecol. 2002;99(1):169170.

4. Usta IM, Hobeika EM, Musa AA, et al. Placenta previa- accreta: risk factors and complications. Am J Obstet Gynecol. 2005;193(3 pt 2):10451049.

5. Wu S, Kocherginsky M, Hibbard JU. Abnormal placentation: twenty-year analysis. Am J ObstetGynecol. 2005;192(5):1458-1461.

6. Mazouni C, Gorincour G, Juhan V, et al. Placenta accreta: a review of current advances in prenatal diagnosis. Placenta. 2007;28(7):599-603.
7. Japaraj RP, Mimin TS, Mukudan K. Antenatal diagnosis of placenta previa accreta in patients with previous cesarean scar. J Obstet Gynecol Res. 2007;33(4):431-437.

8. Esakoff TF, Sparks TN, Kaimal AJ, et al. Diagnosis and morbidity of placenta accreta. Ultrasound Obstet Gynecol. 2011;37(3):324-327.

9. Comstock $\mathrm{CH}$. Antenatal diagnosis of placenta previa accreta: a review. Ultrasound Obstet Gynecol. 2005;26(1):89-96.

10. Warshak CR, Escander R, Hull AD, et al. Accuracy of ultrasonography and magnetic resonance imaging in the diagnosis of placenta accreta. Obstet Gynecol. 2006;108(3 pt 1):573-581.

11. Dwyer BK, Belogolovkin V, Tran L, et al. Prenatal diagnosis of placenta accreta: sonography or magnetic resonance imaging? J Ultrasound Med. 2008;27(9):1275-1281.

12. Chou MM, Ho ESC, Lee YH. Prenatal diagnosis of placenta accreta by transabdominal color doppler ultrasound. Ultrasound Obstet Gynecol. 2000;15(1):28-35.

13. Shih JC, Palacios Jaraquemada JM, Su YN, et al. Role of three-dimensional power Doppler in the antenatal diagnosis of placenta accreta: comparison with gray-scale and color Doppler techniques. Ultrasound Obstet Gynecol. 2009;33(2):193-203.

14. Woodring TC, Klauser CK, Bofill JA, et al. Prediction of placenta accreta by ultrasonography and color doppler imaging. J Matern Fetal Neonatal Med. 2011;24(1):118-121.

15. Thia EW, Lee SL, Tan HK, et al. Ultrasonographical features of morbidly-adherent placentas. Singapore Med J. 2007;48(9):799-802.

16. Hasegawa J, Matsuoka R, Ichizuka K, et al. Predisposing factors for massive hemorrhage during Cesarean section in patients with placenta previa. Ultrasound Obstet Gynecol. 2009;34(1):80-84.

17. Wong HS, Zuccollo J, Tait J, et al. Antenatal topographical assessment of placenta accreta with ultrasound. Aust N Z J Obstet Gynaecol. 2008;48(4):421-423. 